\title{
Suspected Exposure to Filoviruses Among People Contacting Wildlife in Southwestern Uganda
}

\section{Tierra Smiley Evans, ${ }^{1}$ Leonard Tutaryebwa, ${ }^{6}$ Kirsten V. Gilardi, ${ }^{1}$ Peter A. Barry, ${ }^{2}$ Andrea Marzi, ${ }^{5}$ Meghan Eberhardt, ${ }^{2}$ Benard Ssebide, ${ }^{3}$ Michael R. Cranfield, ${ }^{3}$ Obed Mugisha, ${ }^{6}$ Emmanuel Mugisha, ${ }^{6}$ Scott Kellermann, ${ }^{4}$ Jonna A. K. Mazet, ${ }^{1}$ and Christine K. Johnson ${ }^{1}$}

${ }^{1}$ One Health Institute, School of Veterinary Medicine, and ${ }^{2}$ Center for Comparative Medicine, Department of Pathology and Laboratory Medicine, University of California, Davis; and ${ }^{3}$ Gorilla Doctors, Mountain Gorilla Veterinary Project, Inc., Kampala, Uganda; ${ }^{4}$ University of San Francisco, California; ${ }^{5}$ Laboratory of Virology, Division of Intramural Research, National Institute of Allergy and Infectious Diseases, National Institutes of Health, Hamilton, Montana; and ${ }^{6}$ Bwindi Community Hospital, Buhoma, Uganda

Background. Human and filovirus host interactions remain poorly understood in areas where Ebola hemorrhagic fever outbreaks are likely to occur. In the Bwindi region of Uganda, a hot spot of mammalian biodiversity in Africa, human livelihoods are intimately connected with wildlife, creating potential for exposure to filoviruses.

Methods. We tested samples from 331 febrile patients presenting to healthcare facilities near Bwindi Impenetrable Forest, Uganda, by polymerase chain reaction (PCR) analysis and Western blot, using recombinant glycoprotein antigens for Ebola virus (EBOV), Sudan virus (SUDV), Bundibugyo virus (BDBV), and Marburg virus. Behavioral data on contact with wildlife were collected to examine risk factors for filovirus seropositivity.

Results. All patients were negative for active filovirus infection, by PCR analysis. However, patients were seroreactive to SUDV (4.7\%), EBOV (5.3\%), and BDBV (8.9\%), indicating previous exposure. Touching duikers was the most significant risk factor associated with EBOV seropositivity, while hunting primates and touching and/or eating cane rats were significant risk factors for SUDV seropositivity.

Conclusions. People in southwestern Uganda have suspected previous exposure to filoviruses, particularly those with a history of wildlife contact. Circulation of filoviruses in wild animals and subsequent spillover into humans could be more common than previously reported.

Keywords. Filovirus; Ebola virus; Marburg virus; zoonoses; bushmeat; wildlife; Uganda.

Since the discovery of filoviruses 5 decades ago, Ebola hemorrhagic fever (EHF) and Marburg hemorrhagic fever (MHF) outbreaks have become more frequently recognized and pose great risk for regional and international spread [1]. Increased contact between humans and wildlife in regions where wildlife hosts live or migrate is likely contributing to this rise in filovirus outbreaks [2]. Elucidation of human and animal host interactions are needed to institute behavioral changes and risk mitigation aimed at prevention in regions where hemorrhagic fever outbreaks are prone to occur.

Based on field and experimental studies, Pteropodidae fruit bats have been indicated as reservoirs for Marburg virus (MARV) and suggested as possible hosts for Ebola virus (EBOV) [3-5]. Direct exposure to fruit bats through consumption and close proximity (eg, by sharing dwellings and entering mines or caves) has been linked to human EHF and MHF outbreaks in Uganda and other parts of East/Central Africa [6-9]. In addition, MARV and EBOV sequences have been detected in insectivorous bats (Rhinolophus eloquens, Miniopterus inflatus, and

Correspondence: T. Smiley Evans, DVM, PhD, One Health Institute, University of CaliforniaDavis, 1089 Veterinary Medicine Dr, Davis, CA 95616 (tsmevans@ucdavis.edu).

\section{The Journal of Infectious Diseases ${ }^{\circledR} \quad$ 2018;218(S5):S277-86}

(c) The Author(s) 2018. Published by Oxford University Press for the Infectious Diseases Society of America. All rights reserved. For permissions, e-mail: journals.permissions@oup.com. DOl: 10.1093/infdis/jiy251
Hipposideros species), but no links to human EHF and MHF have been reported $[4,10]$. It is unknown whether filoviruses are circulating regularly in bat populations.

Contact with nonhuman primates has also been implicated as a source of human infections with EBOV and Taï Forest virus (TAFV) [11, 12]. However, nonhuman primate species infected with EBOV and TAFV have had severe or fatal illness, suggesting that they are not suitable reservoir species but could potentially transmit filoviruses to humans when hunted and handled after death [12-14]. Members of the Ebolavirus genus have been implicated as one of the causes of the decline of wild common chimpanzees (Pan troglodytes) and western lowland gorillas (Gorilla gorilla gorilla) $[14,15]$ and are considered a major threat to the survival of African great apes [16].

Limited evidence exists for other wild and domestic animal species potentially playing a role in filovirus transmission to either humans and/or other great apes. Duikers (Cephalophus species), small forest antelopes, have been implicated as incidental hosts for EBOV [13]. An increase in brush-tailed porcupine (Atherurus africanus) and wild bush pig (Potamochoerus larvatus) mortality has also temporally and geographically coincided with human EHF outbreaks [17]. In addition, EBOV RNA has been detected in 2 Muridae rodent genera and 1 shrew species (Sylvisorex ollula), although attempts to isolate the virus were unsuccessful [18]. Swine have been confirmed as susceptible domestic animal hosts for Reston virus [19] and 
immunoglobulin G (IgG) EBOV antibodies have been detected in sera from domestic dogs in close proximity to an EBOV epidemic area [20].

Despite multiple pieces of evidence, further investigation and confirmation of potential wildlife reservoirs and additional incidental hosts for filoviruses is lacking, partly due to minimal systematic wildlife surveillance efforts and the availability of accurate diagnostic tests. Challenges which have hampered filovirus surveillance in wildlife include a very short time frame within which to detect viral shedding in animals involved in initial spillover of filoviruses to humans and difficulties in achieving necessary sample sizes in suspected animal reservoirs, particularly among species with unknown population size. Limitations in availability of accurate diagnostic testing include requirement of maximum containment (biosafety level 4) facilities to conduct virus isolation and cross-reactivity between filovirus species among available serological assays. In addition, surveillance and diagnostic capabilities are likely complicated by the presence of unknown filoviruses, which have the potential to cross-react with serological diagnostics and influence immunological susceptibility of reservoir and incidental hosts.

In consideration of these challenges facing wildlife studies, human serological assays optimized for detection of filoviruses, combined with epidemiologic data on human-wildlife contact, could provide valuable insight into the geographic distribution of filoviruses, as well as the wildlife populations in which to focus further studies. Previous human serological studies have linked activities such as scavenging gorilla, chimpanzee, and duiker carcasses to EHF outbreaks in Gabon [12, 13, 21] and have identified forest-dwelling human populations and people living in close proximity to forests in the Central African Republic and the Democratic Republic of Congo (DRC) as high-risk human groups for EBOV exposure [22, 23].

In this study, we investigated potential exposures to filoviruses among acutely febrile patients from the Bakiga and Batwa tribes in southwestern Uganda, and identified possible risk factors for filovirus seropositive status using detailed livelihood and behavior surveys. The Bakiga are agriculturalists, whereas the Batwa were previously hunter-gatherers prior to their displacement from the interior of the Bwindi Impenetrable Forest. The Batwa now live in settlements surrounding Bwindi Impenetrable National Park and have become one of the most marginalized human groups in Uganda. The park dominates this region and is a biodiversity hotspot, harboring among the largest number of primate species of any forest in Africa, including the critically endangered mountain gorilla (Gorilla beringei beringei) [24]. Our study objectives were to (1) identify whether humans in the Bwindi region have had past exposures to filoviruses, by testing for the presence of IgG antibodies to specific filovirus glycoproteins (GPs), and (2) identify wildlife species and types of wildlife interactions associated with human exposure to filoviruses.

\section{METHODS}

\section{Human Subjects}

A total of 331 patients from Bwindi Community Hospital and Byumba Health Center II presenting with fever and accompanying clinical signs were voluntarily enrolled in the study between March and June 2013 (Figure 1). Inclusion criteria included nonspecific signs of viral illness, such as fever (ie, body temperature $>37.8^{\circ} \mathrm{C}$ ), vomiting, diarrhea, abdominal pain, nasal congestion, and/or cough. Blood samples and oropharyngeal swab specimens were collected from each participant. Questionnaires to collect demographic information, travel history, medical history, information on livelihood(s), and interactions with domestic and wild animals were administered in the local language, RunyankoreRukiga. The aim of the study was communicated to all patients or parents/guardians of minors, and written informed consent was obtained from all study participants. Institutional review boards at Makerere University, Bwindi Community Hospital, the University of California-Davis, and the Uganda National Council for Science and Technology approved this study.

\section{Polymerase Chain Reaction (PCR)-Based Diagnostic Techniques}

Total nucleic acid was extracted from whole-blood and oral swab specimens, using the NucliSens MiniMag system (bioMérieux) according to manufacturer's instructions, with the exception of the addition of a 2-hour proteinase $\mathrm{K}$ digestion step with mechanical disruption for whole-blood samples. RNA was reverse transcribed and complementary DNA (cDNA) synthesized using Superscript III First-Strand Synthesis cDNA kits (Invitrogen, Carlsbad, CA). cDNA was analyzed by conventional PCR analysis for the filovirus $\mathrm{L}$ gene, using primers previously designed by Zhai et al [25]. A touchdown protocol was programmed, using a Fast Cycling PCR kit (Qiagen, Germantown, $\mathrm{MD}$ ), with the following reaction conditions: 1 cycle at $95^{\circ} \mathrm{C}$ for 5 minutes; 12 cycles at $96^{\circ} \mathrm{C}$ for 8 seconds, $65^{\circ} \mathrm{C}$ (with a $1^{\circ} \mathrm{C}$ decrease during each subsequent cycle) for $8 \mathrm{sec}$ onds, and $68^{\circ} \mathrm{C}$ for 18 seconds; 35 cycles at $96^{\circ} \mathrm{C}$ for 8 seconds, $52^{\circ} \mathrm{C}$ for 8 seconds, and $68^{\circ} \mathrm{C}$ for 18 seconds; and $1 \mathrm{cycle}$ at $72^{\circ} \mathrm{C}$ for 5 minutes. PCR products of appropriate size were cloned using Topo TA cloning kits (Invitrogen), and sequencing was performed using Sanger dideoxy sequencing at the University of California-Davis DNA sequencing laboratory.

\section{Western Blots}

Samples were $\gamma$-irradiated with a dose of 2 MRad before screening [26]. Samples were denatured in $2.5 \% \beta$-mercaptoethanol, and proteins were separated on $10 \%$ mini-protean TGX gels (Biorad, Hercules, CA) and transferred to Amersham Hybond 0.45 polyvinylidene fluoride membranes (GE-biosciences, Marlborough, MA). Blots were blocked in 5\% nonfat milk and $1 \%$ normal goat serum. Membranes were incubated with serum at a 1:100 dilution overnight at room temperature. Membranes were then incubated with peroxidase-labeled antibody to human 


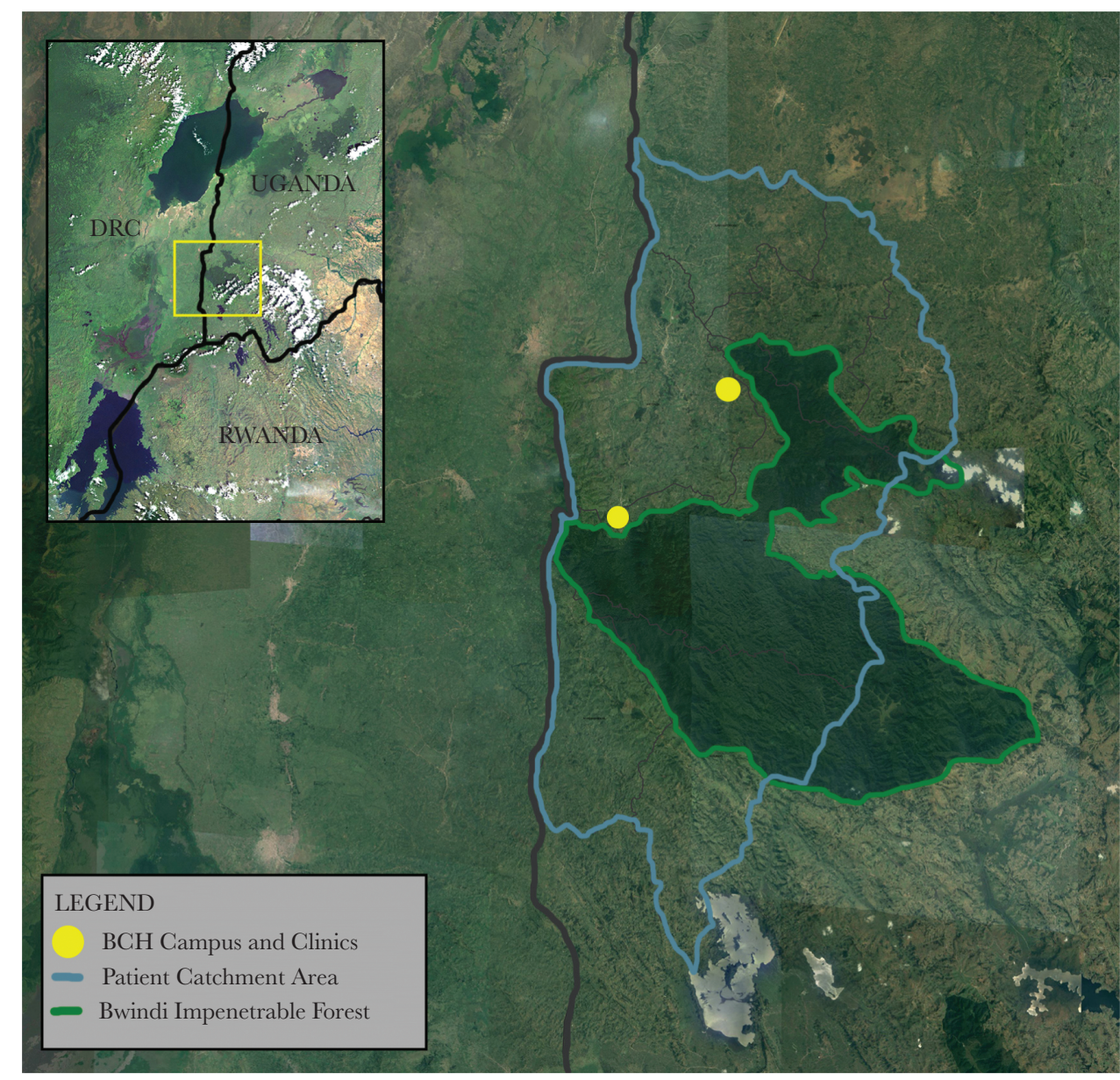

Figure 1. Map of the Bwindi region of southwestern Uganda. Bwindi Community Hospital (BHC) main campus and satellite clinics (yellow) on the edge of the Bwindi Impenetrable Forest. Patients enrolled in the study were residents of Kirundo, Nyabwishenya, Mpungu, Kayonza, Kanungu, Kirima, and Kanyantorogo subcounties in southwestern Uganda.

IgG (KPL, Gaithersburg, MD) at a 1:5000 dilution. The following antigens were used: EBOV, SUDV, and MARV strain Musoke recombinant virus GPs (IBT Bioservices, Gaithersburg, MD) and Bundibugyo virus (BDBV) strain Uganda 2007 recombinant, partial virus envelope GP1 (Ile 33-Gln 304; Acro Biosystems, Newark, DE). Antigens were produced in mammalian cells.

Western blots were validated using convalescent serum from a human who was vaccinated with a recombinant vesicular stomatitis virus (VSV)-based vaccine that expressed an EBOV GP (VSV-EBOV) after suspected EBOV exposure [27], convalescent serum from a MARV-infected nonhuman primate, rabbit anti-SUDV GP polyclonal antibody (IBT Bioservices), and convalescent serum from a rhesus macaque experimentally infected with BDBV [28]. A negative control pool of serum specimens from 20 Ugandan individuals who presented to the Bwindi Community Hospital with respiratory symptoms and showed no filovirus seroreactivity on Western blots, as well as a negative control pool of serum specimens from 5 individuals from other African countries, were used as negative controls. In addition, 15 serum samples collected from volunteer blood donors at the Stanford Blood Center (Palo Alto, CA) were used as negative controls during assay development.

Blots were considered positive for virus if a band of the correct size could be visualized for the $\mathrm{GP}_{1}$ protein of EBOV, SUDV, and MARV (approximately $150 \mathrm{kDa}$; Supplementary Figure 1 and 2) and the partial GP ${ }_{1}$ protein of BDBV (approximately 60 $\mathrm{kDa}$; Supplementary Figure 3) after visual comparison to positive and negative control samples. A conservative approach to interpretation of Western blot band results was taken in that samples were tested in duplicate and were categorized by 2 independent researchers as positive, negative, or indeterminate, based on visualization of appropriate sized bands.

\section{Statistical Analyses}

Individuals with positive and negative samples were used in risk factor analyses and period prevalence calculations; individuals with indeterminate results due to faint bands were excluded (sample sizes used in statistical analyses were as follows: 300 for SUDV analyses, 301 for EBOV analyses, 303 for BDBV analyses, and 331 for MARV analyses). Period prevalence and $95 \%$ binomial exact 
confidence intervals (CIs) were calculated for demographic groups for each filovirus species. When period prevalence was 0 , 1-sided, $97.5 \%$ binomial exact CIs were calculated.

All demographic factors, including age, tribe, and livelihood (Table 1), were evaluated for associations with animal contact behaviors (Tables 2 and 3), using bivariate analyses to assess confounding. Associations between filovirus seropositive status and all individual risk factors in Tables $1-3$ were initially evaluated by the 2 -sided Fisher exact test or the $\chi^{2}$ test as appropriate, and associations were measured by odds ratios (ORs). Multiple exposures were common, and some behavior categories were associated with each other; therefore, adjustment for various risk factors in multivariable analyses was done sequentially, with priority given to those with a higher OR on crude analysis, those that were highly significant, and those factors with previous evidence based on other investigations.

Multivariable logistic regression was then used to assess the association of risk factors with filovirus exposure for variables that were significant on bivariate analysis $(P<.1)$. Separate logistic models were generated for each filovirus species to account for variation in wildlife reservoirs and the risk of transmission to susceptible people. Multivariable logistic regression was also used to assess the association between demographic and livelihood factors and wild animal contact. Variables were included if they significantly improved model fit, based on the likelihood ratio test $(P<.1)$, while minimizing the Akaike information criterion. Overall model fit was assessed using the Hosmer-Lemeshow goodness-of-fit test [29]. All statistical analyses were performed using Stata (StataCorp, College Station, TX). Network analyses to generate a wildlife taxa-interface network display (Figure 2) were conducted in the network analysis platform Gephi, using the force-directed algorithm ForceAtlas2 [30].

\section{Ethical Standards}

All procedures contributing to this work complied with the ethical standards of the relevant national and institutional committees on human experimentation and with the Helsinki Declaration of 1975, as revised in 2008.

Table 1. Sudan Virus (SUDV), Ebola Virus (EBOV), and Bundibugyo virus (BDBV) Prevalence, by Demographic Characteristic, Among Individuals With Wildlife Contact Who Presented to Healthcare Facilities in the Bwindi Region of Uganda During March-June 2013

\begin{tabular}{|c|c|c|c|c|c|c|c|c|c|}
\hline \multirow[b]{2}{*}{ Characteristic } & \multicolumn{3}{|c|}{ SUDV } & \multicolumn{3}{|c|}{ EBOV } & \multicolumn{3}{|c|}{ BDBV } \\
\hline & No. Pos & No. Neg & $\begin{array}{l}\text { Period Prevalence } \\
\qquad\left(95 \% \mathrm{Cl}^{\mathrm{a}}\right)\end{array}$ & No. Pos & No. Neg & $\begin{array}{l}\text { Period Prevalence } \\
\quad\left(95 \% \mathrm{Cl}^{\mathrm{a}}\right)\end{array}$ & No. Pos & No. Neg & $\begin{array}{l}\text { Period Prevalence } \\
\qquad\left(95 \% \mathrm{Cl}^{\mathrm{a}}\right)\end{array}$ \\
\hline \multicolumn{10}{|l|}{ Ethnic group } \\
\hline Bakiga & 11 & 267 & $3.96(1.99-6.97)$ & 14 & 263 & $5.05(2.79-8.33)$ & 23 & 261 & $8.10(5.21-11.90)$ \\
\hline Batwa & 2 & 12 & $14.29(1.80-42.81)$ & 1 & 14 & $6.67(.17-31.95)$ & 2 & 11 & $15.38(1.92-45.45)$ \\
\hline \multicolumn{10}{|l|}{ Sex } \\
\hline Male & 7 & 133 & $5.00(2.03-10.03)$ & 9 & 130 & $6.67(3.00-11.94)$ & 12 & 129 & 8.51 (4.47-14.39) \\
\hline Female & 7 & 142 & $4.70(1.9-9.43)$ & 7 & 143 & $4.67(1.90-9.38)$ & 13 & 137 & 8.67 (4.70-14.39) \\
\hline \multicolumn{10}{|l|}{ Age group } \\
\hline $0-9$ & 1 & 32 & $3.03(.08-15.76)$ & 1 & 31 & $3.13(.07-16.22)$ & 5 & 25 & $16.67(5.64-34.72)$ \\
\hline $10-19$ & 2 & 62 & $3.13(.38-10.83)$ & 5 & 58 & $7.94(2.63-17.56)$ & 10 & 55 & $15.38(7.63-26.48)$ \\
\hline $20-29$ & 3 & 58 & $4.92(1.03-13.71)$ & 2 & 60 & $3.23(.39-11.17)$ & 2 & 62 & $3.13(.38-10.48)$ \\
\hline $30-39$ & 2 & 41 & $4.65(.57-15.81)$ & 2 & 42 & $4.55(.55-15.47)$ & 1 & 44 & $2.22(.06-11.77)$ \\
\hline $40-49$ & 0 & 24 & $0.00^{b}(.00-14.25)$ & 3 & 20 & $13.04(2.78-33.59)$ & 1 & 25 & $3.85(.09-19.63)$ \\
\hline $50-59$ & 2 & 22 & $8.33(1.03-27.00)$ & 3 & 24 & $11.11(2.35-29.16)$ & 0 & 26 & $0.00^{b}(.00-13.23)$ \\
\hline $60-69$ & 0 & 15 & $0.00^{b}(.00-21.80)$ & 0 & 17 & $0.00^{b}(.00-19.51)$ & 1 & 16 & $5.88(.15-28.69)$ \\
\hline$\geq 70$ & 1 & 14 & $6.67(.17-31.95)$ & 0 & 15 & $0.00^{\mathrm{b}}(.00-21.80)$ & 4 & 10 & $28.57(8.39-58.10)$ \\
\hline \multicolumn{10}{|l|}{ Primary occupation } \\
\hline Housework & 12 & 224 & $5.08(2.65-8.71)$ & 13 & 224 & $5.49(2.95-9.20)$ & 19 & 227 & $7.72(4.71-11.80)$ \\
\hline Crop farming & 12 & 228 & $5.00(2.61-8.57)$ & 14 & 228 & $5.79(2.89-9.01)$ & 19 & 230 & $7.63(4.66-11.65)$ \\
\hline Livestock farming & 9 & 196 & $4.39(2.03-8.07)$ & 11 & 193 & $5.39(2.72-9.44)$ & 14 & 197 & $6.64(3.67-10.88)$ \\
\hline Hunting & 1 & 0 & $100.00(.00-97.50)$ & 0 & 1 & $0.00^{\mathrm{b}}(.00-97.50)$ & 0 & 1 & $0.00^{b}(.00-97.50)$ \\
\hline Working in BINP & 0 & 5 & $0.00^{b}(.00-52.18)$ & 0 & 5 & $0.00^{b}(.00-52.18)$ & 0 & 5 & $0.00^{b}(.00-52.18)$ \\
\hline Trading & 1 & 4 & $20.00(.51-71.64)$ & 0 & 5 & $0.00^{b}(.00-52.18)$ & 0 & 5 & $0.00^{\mathrm{b}}(.00-52.18)$ \\
\hline Business & 0 & 6 & $0.00^{b}(.00-45.93)$ & 0 & 6 & $0.00^{\mathrm{b}}(.00-45.93$ & 0 & 6 & $0.00^{\mathrm{b}}(.00-45.93)$ \\
\hline Overall & 14 & 286 & $4.67(2.57-7.71)$ & 16 & 285 & $5.32(3.06-8.46)$ & 27 & 276 & $8.91(5.95-12.70)$ \\
\hline
\end{tabular}

Indeterminate test results were excluded from analyses.

Abbreviation: BINP, Bwindi Impenetrable National Park; Cl, confidence interval; Neg, negative; Pos, positive.

${ }^{a}$ Data are $95 \%$ binomial exact $\mathrm{Cls}$, unless otherwise indicated.

bOne-sided $97.5 \%$ binomial exact $\mathrm{Cls}$ were calculated. 


\begin{tabular}{|c|c|c|c|c|}
\hline Risk Factor ${ }^{a}$ & $\begin{array}{c}\text { Seroprevalence in Exposed Group, } \\
\text { Proportion (\%) }\end{array}$ & $\begin{array}{c}\text { Seroprevalence in Nonexposed Group, } \\
\text { Proportion (\%) }\end{array}$ & OR $(95 \% \mathrm{Cl})$ & $P$ \\
\hline \multicolumn{5}{|l|}{ Consumed wildlife ${ }^{b}$} \\
\hline Primate & $1 / 8(12.5)$ & $15 / 285(5.3)$ & $2.57(.30-22.27)$ & .365 \\
\hline Bat & $\ldots$ & $\ldots$ & $\ldots$ & $>.999$ \\
\hline Rodent & 2/13 (15.4) & $14 / 282(5.0)$ & $3.48(.70-17.23)$ & .152 \\
\hline Duiker & 6/72 (8.3) & $10 / 223(4.5)$ & $1.94(.68-5.53)$ & .217 \\
\hline Porcupine $^{c}$ & 1/7 (18.7) & $15 / 288(5.2)$ & $3.03(.34-26.83)$ & .326 \\
\hline Wild pig & 6/63 (9.5) & 10/232 (4.3) & $2.34(.82-6.70)$ & .114 \\
\hline Hippopotamus & $4 / 57(7.0)$ & 10/193 (5.2) & $1.35(.30-4.92)$ & .618 \\
\hline Civet & $\ldots$ & $\ldots$ & $\ldots$ & $>.999$ \\
\hline Any & $11 / 115(9.6)$ & $5 / 180(2.8)$ & $\ldots$ & \\
\hline \multicolumn{5}{|c|}{ Contacted wildlife that raided crops } \\
\hline Primate & $3 / 68(4.4)$ & $11 / 183(6.0)$ & $0.73(.13-2.89)$ & .765 \\
\hline Bat & $1 / 12(8.3)$ & $13 / 239(5.4)$ & $1.53(.03-11.97)$ & .514 \\
\hline Rodent (all species) & $14 / 223(6.3)$ & $0 / 28(0.0)$ & $\ldots$ & $>.999$ \\
\hline Rodent (cane rat) & $0 / 1(0.0)$ & 14/253 (5.5) & $\ldots$ & $>.999$ \\
\hline Duiker & $1 / 5(20.0)$ & $13 / 246(5.3)$ & $3.78(.07-37.33)$ & .280 \\
\hline Porcupine $^{c}$ & $1 / 2(50.0)$ & $13 / 249(5.2)$ & $9.57(.15-191.09)$ & .151 \\
\hline Wild pig & $1 / 5(20.0)$ & 13/246 (5.3) & $3.78(.07-37.33)$ & .280 \\
\hline Civet & $\ldots$ & $\ldots$ & $\ldots$ & $>.999$ \\
\hline Any & $14 / 233(6.0)$ & 0/18 (0.0) & $\ldots$ & \\
\hline \multicolumn{5}{|l|}{ Touched/handled wildlife } \\
\hline Primate & 0/17 (0.0) & $16 / 279(5.7)$ & $\ldots$ & $>.999$ \\
\hline Bat & $0 / 1(0.0)$ & 16/295 (5.4) & $\ldots$ & $>.999$ \\
\hline Rodent (all species) & $15 / 210(7.1)$ & $1 / 86(1.2)$ & $6.14(.91-261.51)$ & .049 \\
\hline Rodent (cane rat) & $1 / 6(16.7)$ & 13/245 (5.3) & $3.14(.06-28.90)$ & .319 \\
\hline Duiker & 3/16 (18.8) & $13 / 280(4.6)$ & $4.03(.67-16.83)$ & .065 \\
\hline Porcupine $^{c}$ & $1 / 6(16.7)$ & $15 / 290(5.2)$ & $3.22(.06-29.19)$ & .311 \\
\hline Wild pig & $0 / 4(0.0)$ & 16/292 (5.5) & $\ldots$ & $>.999$ \\
\hline Civet & 0/1 (0.0) & 16/295 (5.4) & $\ldots$ & $>.999$ \\
\hline Any & $15 / 212(7.1)$ & $1 / 84(1.2)$ & $\ldots$ & \\
\hline \multicolumn{5}{|c|}{ Contacted wildlife in forest } \\
\hline Primate & 0/3 (0.0) & 16/292 (5.5) & $\ldots$ & $>.999$ \\
\hline Bat & $0 / 1(0.0)$ & 16/294 (5.4) & $\ldots$ & $>.999$ \\
\hline Rodent & $1 / 8(12.5)$ & $15 / 287(5.2)$ & $2.39(.05-19.88)$ & .382 \\
\hline Wild birds $^{d}$ & $2 / 3(66.7)$ & $12 / 248(4.8)$ & $13.78(1.03-128.96)$ & .024 \\
\hline Duiker & $0 / 2(0.0)$ & 16/293 (5.5) & $\ldots$ & $>.999$ \\
\hline Porcupine $^{c}$ & $\ldots$ & $\ldots$ & $\ldots$ & \\
\hline Wild pig & $0 / 2(0.0)$ & 16/293 (5.5) & $\ldots$ & $>.999$ \\
\hline Civet & $0 / 1(0.0)$ & 16/294 (5.4) & $\ldots$ & $>.999$ \\
\hline Any & 2/14 (14.3) & $12 / 237(5.1)$ & $\ldots$ & \\
\hline
\end{tabular}

Abbreviations: $\mathrm{Cl}$, confidence interval; OR, odds ratio.

a Having wildlife in homes, having wildlife near homes, having wildlife close to water source, sharing fruits/vegetables with wildlife, being injured by wildlife, finding dead wildlife, hunting wildlife, and consuming wildlife were not significantly associated with EBOV seropositivity for any wildlife species.

bIncludes both current and past wildlife consumption.

${ }^{c}$ Porcupines are reported separately from rodents because porcupines were not considered to be in the same animal category as smaller rodent species by local study participants

${ }^{d}$ Contact with wild birds, while significant on bivariate analysis, did not have a large enough sample size to evaluate as a risk factor in a multivariable analysis.

\section{RESULTS}

\section{Detection of Previous Exposure to SUDV, EBOV, and BDBV}

Febrile patients in this study did not exhibit any signs of hemorrhagic fever and no patients reported having suffered a hemorrhagic illness previously. Diagnoses provided by medical facilities included, malaria, pneumonia, typhoid fever, respiratory infection, urinary tract infection, gastritis, brucellosis, and tuberculosis. All patients had negative results on consensus
PCR tests of blood and oral swab specimens for detection of filoviruses. Among study participants, 14 of 300 were seropositive for SUDV, 16 of 301 were seropositive for EBOV, and 27 of 303 were seropositive for BDBV by conventional Western blot (Table 1). Seropositivity to MARV was not detected. One sample was positive for both EBOV and SUDV, and 1 was positive for both EBOV and BDBV. Serologic results for SUDV, EBOV, and BDBV were not significantly correlated with each 
Table 3. Results of Bivariate Analyses for Risk Factors for Exposure to Sudan Virus (SUDV)

\begin{tabular}{|c|c|c|c|c|}
\hline Risk Factor ${ }^{a}$ & $\begin{array}{c}\text { Seroprevalence in Exposed Group, } \\
\text { Proportion (\%) }\end{array}$ & $\begin{array}{c}\text { Seroprevalence in Nonexposed Group, } \\
\text { Proportion (\%) }\end{array}$ & OR (95\% Cl) & $P$ \\
\hline \multicolumn{5}{|l|}{ Hunted wildlife } \\
\hline Primate & 4/7 (57.1) & 8/242 (3.3) & $39.0(7.46-204.00)$ & .001 \\
\hline Bat & $0 / 1(0.0)$ & $12 / 248(4.8)$ & $\ldots$ & $>.999$ \\
\hline Rodent & 1/3 (33.3) & $11 / 246(4.5)$ & $7.46(.13-100.13)$ & .173 \\
\hline Duiker & $1 / 3(33.3)$ & $11 / 246(4.5)$ & $7.46(.13-100.13)$ & .173 \\
\hline Porcupine ${ }^{b}$ & $0 / 1(0.0)$ & $12 / 248(4.8)$ & $\ldots$ & $>.999$ \\
\hline Wild pig & 1/3 (33.3) & $11 / 246(4.5)$ & $7.46(.13-100.13)$ & .173 \\
\hline Civet & $\ldots$ & $\ldots$ & $\ldots$ & \\
\hline Any & $4 / 9(44.4)$ & $8 / 240$ (3.3) & $\ldots$ & \\
\hline \multicolumn{5}{|l|}{ Consumed wildlife ${ }^{c}$} \\
\hline Primate & $0 / 6(0.0)$ & $13 / 288(4.5)$ & $\ldots$ & $>.999$ \\
\hline Bat & $\ldots$ & $\ldots$ & $\ldots$ & \\
\hline Rodent (all species) & $3 / 14(21.4)$ & 10/280 (3.6) & $6.00(.95-26.81)$ & .029 \\
\hline Rodent (cane rat) & $3 / 11(27.3)$ & 9/237 (4.0) & $7.18(1.08-34.20)$ & .021 \\
\hline Duiker & $5 / 70(7.1)$ & $8 / 224$ (3.6) & $2.00(.50-7.18)$ & .229 \\
\hline Porcupine ${ }^{b}$ & 1/7 (14.3) & $12 / 287(4.2)$ & $3.42(.07-30.21)$ & .296 \\
\hline Wild pig & $4 / 64(6.25)$ & 9/230 (3.9) & $1.60(.35-5.93)$ & .494 \\
\hline Hippopotamus & $4 / 60(6.67)$ & 8/188 (4.3) & $1.57(.33-6.09)$ & .491 \\
\hline Civet & $0 / 1(0.0)$ & $13 / 293(4.4)$ & $\ldots$ & $>.999$ \\
\hline Any & $5 / 115$ (4.3) & $8 / 179$ (4.5) & $\ldots$ & \\
\hline \multicolumn{5}{|c|}{ Contacted wildlife that raided crops } \\
\hline Primate & 6/71 (8.5) & 6/178 (3.4) & $2.51(.64-9.68)$ & .111 \\
\hline Bat & $0 / 12(0.0)$ & $12 / 237(5.1)$ & $\ldots$ & $>.999$ \\
\hline Rodent (all species) & $11 / 222(5.0)$ & $1 / 27(3.7)$ & $1.34(.18-59.68)$ & $>.999$ \\
\hline Rodent (cane rat) & $1 / 2(50.0)$ & $11 / 249(4.4)$ & $11.31(.17-227.25)$ & .131 \\
\hline Duiker & $0 / 5(0.0)$ & $12 / 244(4.9)$ & $\ldots$ & $>.999$ \\
\hline Porcupine ${ }^{b}$ & $0 / 3(0.0)$ & $12 / 246(4.9)$ & $\ldots$ & $>.999$ \\
\hline Wild pig & $1 / 5(20.0)$ & $11 / 244(4.4)$ & $4.43(.09-44.54)$ & .248 \\
\hline Civet & $\ldots$ & $\ldots$ & $\ldots$ & \\
\hline Any & $11 / 232(4.7)$ & $1 / 17(5.9)$ & $\ldots$ & \\
\hline \multicolumn{5}{|l|}{ Touched/handled wildlife } \\
\hline Primate & 3/16 (18.8) & $10 / 278(3.6)$ & $5.21(.83-22.89)$ & .039 \\
\hline Bat & $\ldots$ & $\ldots$ & $\ldots$ & \\
\hline Rodent (all species) & $11 / 212(5.2)$ & $2 / 82(2.4)$ & $2.13(.48-20.12)$ & .526 \\
\hline Rodent (cane rat) & $2 / 7(28.6)$ & $10 / 241(4.1)$ & $6.89(.61-42.55)$ & .056 \\
\hline Duiker & $1 / 14(7.1)$ & $12 / 280(4.3)$ & $1.67(.04-12.87)$ & .486 \\
\hline Porcupine ${ }^{b}$ & $1 / 6(16.7)$ & $12 / 288(4.2)$ & $4.0(.08-37.09)$ & .264 \\
\hline Wild pig & $0 / 4(0.0)$ & $13 / 290(4.5)$ & $\ldots$ & $>.999$ \\
\hline Civet & $0 / 1(0.0)$ & 13/293 (4.4) & $\ldots$ & $>.999$ \\
\hline Any & $11 / 213(5.2)$ & 2/81 (2.5) & $\ldots$ & \\
\hline
\end{tabular}

Abbreviations: $\mathrm{Cl}$, confidence interval; OR, odds ratio.

aHaving wildlife in homes, having wildlife near homes, having wildlife close to water source, contacting wildlife in the forest, sharing fruits/vegetables with wildlife, being injured by wildlife, and finding wildlife dead were not significantly associated with SUDV seropositivity for any wildlife species.

${ }^{b}$ Porcupines are reported separately from rodents because porcupines were not considered to be in the same animal category as smaller rodent species by local study participants.

Includes both current and past wildlife consumption.

other $\left(\mathrm{R}^{2}=0.03\right.$ for the correlation between SUDV and EBOV, $\mathrm{R}^{2}=-0.002$ for the correlation between EBOV and BDBV, and $\mathrm{R}^{2}=0.002$ for the correlation between SUDV and BDBV), indicating that there was limited cross-reactivity among known filovirus species on these serological assays.

\section{Wild Animal Contact in Study Community}

Over ninety-nine percent of patients reported some form of contact with wildlife. The wildlife species most commonly contacted were rodents (Rodentia species; 99.6\% had indirect contact, and $86.4 \%$ had direct contact), followed by primates (Primate species; $62.3 \%$ and $15.5 \%$, respectively), bats (Chiroptera species; $67.0 \%$ and $5.5 \%$, respectively), duiker (Cephalophus species, 5.5\% and 29.0\%, respectively), wild pig (Potamochoerus larvatus; $8.1 \%$ and $26.6 \%$, respectively), porcupine (Atherurus africanus; $3.3 \%$ and $4.1 \%$, respectively), and civet (Civettictis civetta; $0.7 \%$ and $0.7 \%$, respectively). Contact due to the presence of wild animals in and around peoples' 


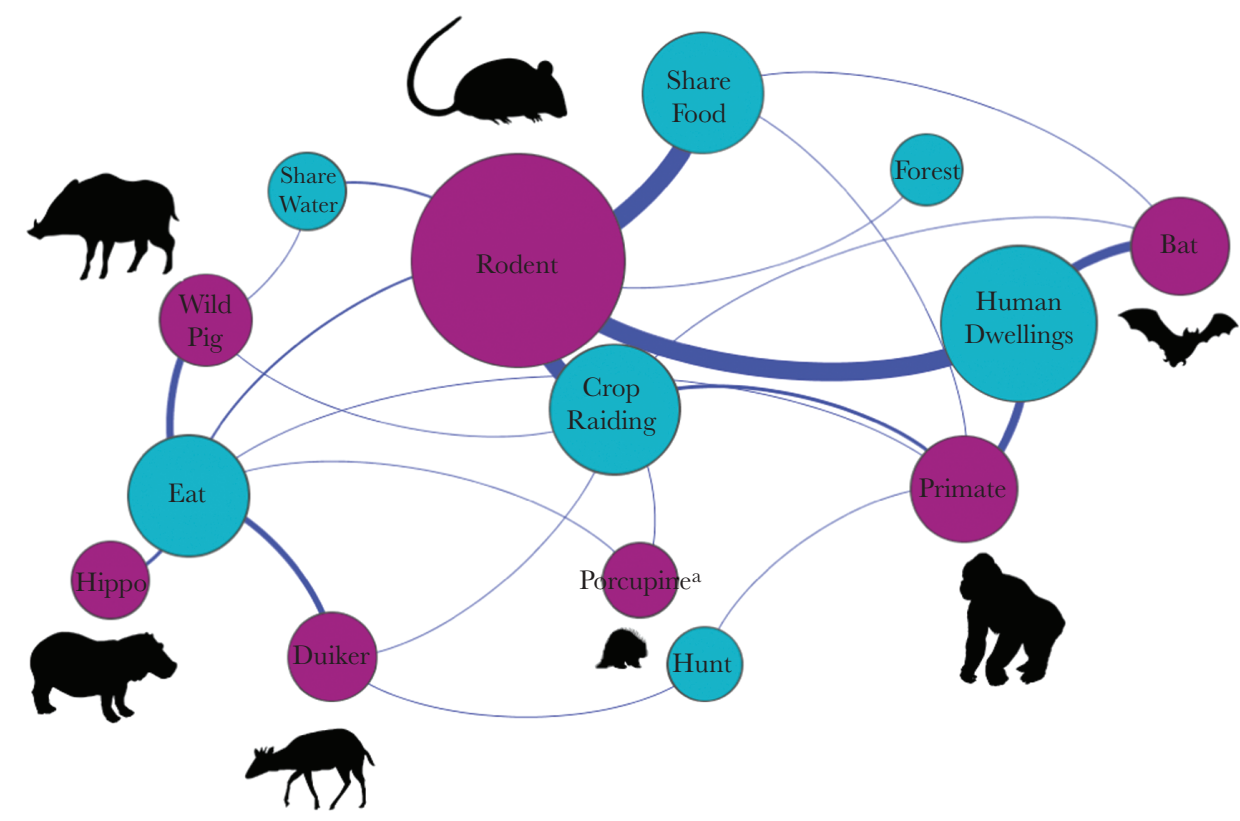

Figure. 2. Epidemiologic bipartite network map showing disease transmission interfaces shared by humans and wildlife in the Bwindi region of southwestern Uganda. For transmission interfaces (blue), the node size is proportionate to the number of people reporting direct or indirect contact with wildlife at each interface. For wildlife taxa (purple), the node size is proportionate to the number of people reporting contact with each respective wildlife taxa. Highly connected and more-central interfaces represent greater opportunities for contact with multiple wildlife host taxa and therefore present greater chances for humans to encounter zoonotic pathogens. This network was created using data on contact between humans and animals that were collected from 331 febrile individuals presenting to medical facilities in the Bwindi region. aPorcupines are reported separately from rodents because porcupines were not considered to be in the same animal category as smaller rodent species by local study participants.

homes was widespread (99\% [270 of 274 patients]), and contact with animals that were raiding crops (93\% [255 of 274]) and sharing food items (91\% [296 of 325]) was also common. Contact with animals through bushmeat hunting was less commonly reported (5\% [13 of 274 patients]; Figure 2).

On multivariable analysis, men were more likely than women to consume wildlife (OR, 3.18; 95\% CI, 1.09-9.32; $P=.035$ ) and to touch duikers (OR, 6.62; 95\% CI, 1.55-28.21; $P=.011)$. People working as park personnel were 9.5 times as likely to consume wildlife than people with other occupations (95\% CI, 1.43-63.25; $P=.020$ ). People of the Batwa tribe were also 9.7 times as likely to consume wildlife (95\% CI, 2.08-44.84; $P=.004), 7.1$ times as likely to hunt wild animals (95\% CI, $1.20-41.55 ; P=.031$ ), and 9.5 times as likely to touch a duiker $(95 \% \mathrm{CI}, 1.71-52.76 ; P=.010)$ than people of the Bakiga tribe.

\section{Wildlife Contact Associated With EBOV Seropositivity}

Results of our multivariable model showed that people with a history of touching duikers were 5.6 times as likely to be seropositive for EBOV than people who reported no contact with duikers (95\% CI, 1.23-25.17; $P=.026$; Table 2 and Table 4). Age group was included in the model because a history of touching duikers was found to increase with age. The log odds of EBOV seropositivity was predicted by use of the following logistic model: $\ln [P(x) / 1-P(x)]=-2.64-0.11(A)+1.72\left(T_{d}\right)$, where $P(x)$ denotes the probability of a person being seropositive for
EBOV (Hosmer-Lemeshow model goodness-of-fit $\chi^{2}=76.38$; $P=.785), A$ denotes age in years, and $T_{d}$ denotes a history of touching a duiker.

\section{Wildlife Contact Associated With SUDV Seropositivity}

Results of our multivariable model showed that people reporting a history of hunting primates were 37.5 times as likely to have evidence of past exposure to SUDV than people who did not report hunting primates (95\% CI, 6.20-226.41; $P<.001$; Table 4). Also, people with a past history of touching or eating cane rats (Thryonomys swinderianus) were 10.7 times as likely to show serologic evidence for past exposure to SUDV than people who did not report touching or eating cane rats (95\% CI, 1.95-58.15; $P=.006$; Table 4). Tribal affiliation was included in the model because being of Batwa ethnicity was significantly associated with hunting wild animals. On bivariate analyses, both touching and eating cane rats were independently associated with SUDV seropositivity (Table 3) but, when combined, were a better predictor of risk of exposure, and they were therefore incorporated as a combined variable into the multivariable model. The log odds of SUDV seropositivity was predicted by use of the following logistic model: $\ln [P(x) / 1-P(x)]=-3.81+1.79(T)+2.37\left(C_{t e}\right)+3.62\left(P_{h}\right)$, where $P(x)$ denotes the probability of a person being seropositive for SUDV (Hosmer-Lemeshow model goodness of fit $\chi^{2}=0.42$; $P=.810), T$ denotes tribe, $C_{t e}$ denotes a history of touching or eating a cane rat, and $P_{h}$ denotes a history of hunting primates. 


\begin{tabular}{|c|c|c|c|c|}
\hline Filovirus, Risk Factor & $\begin{array}{l}\text { Seroprevalence of Risk Factor, } \\
\text { Exposed Group, Proportion (\%) }\end{array}$ & $\begin{array}{c}\text { Seroprevalence of Risk Factor, } \\
\text { Nonexposed Group, Proportion (\%) }\end{array}$ & OR $(95 \% \mathrm{Cl})$ & $P$ \\
\hline \multicolumn{5}{|l|}{ Ebola virus ${ }^{a}$} \\
\hline Touched duiker & 3/16 (18.8) & $13 / 280(4.6)$ & $5.57(1.23-25.17)$ & .026 \\
\hline \multicolumn{5}{|l|}{ Sudan virus ${ }^{b}$} \\
\hline Hunted primate & $4 / 7(57.1)$ & $8 / 242$ (3.3) & $37.47(6.20-226.41)$ & $<.001$ \\
\hline Touched/consumed cane rat & $3 / 12(25.0)$ & $9 / 235$ (3.8) & $10.65(1.95-58.15)$ & .006 \\
\hline
\end{tabular}

${ }^{a}$ Age was associated with touching a duiker. Therefore, age was included in the final multivariable model.

${ }^{b}$ Being of the Batwa tribe was associated with hunting primates. Therefore, tribe was included in the final multivariable model.

No variables describing contact between humans and wildlife were significant on bivariate analysis for BDBV.

\section{DISCUSSION}

We provide evidence for previous exposure to filoviruses among people in the Bwindi region of southwestern Uganda. Our serologic findings fill a gap in geographic exposure data for the region, as outbreaks of EHF have occurred in neighboring districts of Bundibugyo and Kibaale and across the border in the DRC [9, 31-33], and MHF has occurred in Kabale and Maramagambo forests $[9,34,35]$.

It is unlikely that clinically significant EHF cases have occurred and gone unreported in the Bwindi region, given the accessibility of Bwindi Community Hospital and its associated satellite clinics. Additionally, cases of filovirus-associated hemorrhagic fever in mountain gorillas, a species heavily monitored, highly contacted by people, and susceptible to filovirus infection, have not been reported. Identification of IgG antibodies to filovirus GPs in humans presenting to clinics in Bwindi therefore could be due to asymptomatic infection with SUDV, EBOV, or BDBV and/or infection with serologically cross-reactive low-pathogenicity or nonpathogenic undiscovered filoviruses that also have wildlife hosts.

Previous studies suggest that EBOV infections can by asymptomatic or minimally symptomatic [36-38]. Seropositivity in people who did not report a history of hemorrhagic fever has been demonstrated in EHF-endemic regions [23, 39], and asymptomatic family members of patients infected with known filovirus species have shown seropositivity [40]. We also cannot rule out the possibility that we detected antigenically similar but as-yet unknown filoviruses. Additional filoviruses are expected to be discovered as diagnostic assays and surveillance activities improve. Past serosurveys using antigens that are more broadly reactive among filoviruses have detected filovirus exposure among humans and wildlife living in areas where no cases of EHF have ever been reported, such as the Aka population of the Central African Republic [22] and fruit bat populations in Bangladesh [41]. We used GP antigens in this study because it is predicted that the antibody response to GP is more virus specific, owing to the larger genetic variability with this protein
[42]. Serologic cross-reactivity between filovirus species has however been noted in enzyme-linked immunosorbent assays using recombinant GP antigens, although the highest reactions were uniformly detected against the GP antigen homologous to the correct virus species [43].

Interestingly, no one tested positive for a previous exposure to MARV, even though outbreaks of MARV infection have occurred in close proximity to the Bwindi region. An outbreak of MARV infection occurred in 2012 in Kabale District [44], approximately $26 \mathrm{~km}$ from the southern corner of Bwindi Impenetrable Forest. In addition, 2 foreigners who visited caves in Maramagambo Forest in the southern corner of Queen Elizabeth National Park, approximately $80 \mathrm{~km}$ from the northern-most corner of Bwindi Impenetrable Forest, exported MARV in 2008 to the United States and the Netherlands $[45,46]$. MARV has a documented case-fatality rate between $24 \%-88 \%$, indicating that there is potential for detection of seropositive survivors $[47,48]$. Our lack of detection of MARVseropositive people in the Bwindi region suggests that exposure to MARV could be rarer than for other filoviruses and that wildlife reservoirs are more restricted in range or habitat.

We found variations in seroprevalence among people engaged in different activities involving wildlife, providing epidemiologic evidence that filoviruses circulating in Bwindi are of wildlife origin. In contrast to similar studies in West and Central Africa, where bushmeat hunting is the major activity leading to human and wild animal contact, we found a broader scope of activities that leads to interaction with wildlife.

Contact with duiker was identified as a risk factor for EBOV exposure. Duikers are frequently eaten by humans in many parts of equatorial Africa and have been previously implicated as incidental hosts for EBOV. Duikers are believed to become infected with EBOV through scavenging nonhuman primate carcasses [13], an activity that is plausible in Bwindi given the prevalence and diversity of nonhuman primate species. Duikers are the most common animal caught in snares in Bwindi Impenetrable National Park, and people directly contact this species through hunting, food preparation, and consumption. Duikers also frequently leave the forest and can be found crop raiding among private gardens. 
Hunting primates was identified as a significant risk factor for SUDV seropositivity, consistent with previous findings, which have linked outbreaks of EHF, caused by EBOV, to contact with dead common chimpanzees and/or western lowland gorillas [12, 13]. Interestingly, contrary to previous beliefs that hunting and consumption of primates was almost nonexistent in Uganda [24], we identified several people presenting to Bwindi Community Hospital who reported hunting primates or knowing others in their close community who hunted primates. All hunting of vertebrate species living within Ugandas forest reserves is prohibited by law, and Ugandans generally consider primate meat consumption culturally unacceptable. Hunting of primates, however, may be on the increase in this region because of population growth both within Uganda and through influx of refugees from the DRC, where primate bushmeat consumption is more common.

Contact with cane rats was also identified as a risk factor for SUDV exposure. Rodents have been identified as potential filovirus hosts in previous investigations, but evidence has been weak and they have received less attention as more evidence has pointed toward bats and primates as reservoirs and incidental hosts, respectively. Cane rats are of particular concern as a potential host for infectious diseases because of their proximity to people. They commonly range freely around human dwellings and are also raised as a source of meat in West and Central Africa. In the Bwindi region, cane rats are one of the most commonly eaten wildlife species and are frequently found raiding crops and food storage areas, where people indirectly contact their feces and/or urine.

Finding evidence for filoviruses in Bwindi could have implications not only for the human communities surrounding and using the park but also for the critically endangered mountain gorilla. Human and mountain gorilla health and livelihoods in this region are intimately connected; they are susceptible to the same pathogens, and gorilla tourism supports the local economy, which in turn preserves the forest. A filovirus hemorrhagic fever outbreak in Bwindi could have devastating implications for humans and gorillas alike. Additionally, if unknown filoviruses are circulating in Bwindi, which theoretically could confer some degree of cross-protective immunity, this might help explain why filovirus-associated hemorrhagic fever outbreaks have not been reported to date in this region. A better understanding of the filoviruses circulating in Bwindi, as well as their hosts and/or likely reservoir species, is needed to recognize and better understand any potential threat. We conclude that broader surveillance strategies are needed, moving beyond dead nonhuman primates and the bat species tested to date, to characterize filovirus transmission cycles in Africa.

\section{Supplementary Data}

Supplementary materials are available at The Journal of Infectious Diseases online. Consisting of data provided by the authors to benefit the reader, the posted materials are not copyedited and are the sole responsibility of the authors, so questions or comments should be addressed to the corresponding author.

\section{Notes}

Acknowledgments. We thank the physicians, nurses, diagnosticians, and staff of the Bwindi Community Hospital and Byumba Health Center II, for facilitating this project; Heinz Feldmann, for training and supplying positive control sera; Tracey Goldstein, Brett Smith, Jasmine Pante, and Alex Tremeau-Bravard, for project advice and laboratory support; and Steven Soe Evans, for manuscript editing.

Disclaimer. The contents are the responsibility of the authors and do not necessarily reflect the views of the US Agency for International Development, the National Institutes of Health, or the US government.

Financial support. This work was supported by the William J. Fulbright Foundation, the US Agency for International Development (USAID) Emerging Pandemic Threats PREDICT project (grant GHNA-009-00010-00), and the Intramural Research Program of the National Institute of Allergy and Infectious Diseases, National Institutes of Health.

Potential conflicts of interest. All authors: No reported conflicts of interest. All authors have submitted the ICMJE Form for Disclosure of Potential Conflicts of Interest. Conflicts that the editors consider relevant to the content of the manuscript have been disclosed.

\section{References}

1. Centers for Disease Control. 2014 Ebola outbreak in West Africa. http://www.cdc gov/vhf/ebola/outbreaks/2014-west-africa/. Accessed 15 January 2017.

2. Muyembe-Tamfum JJ, Mulangu S, Masumu J, Kayembe JM, Kemp A, Paweska JT. Ebola virus outbreaks in Africa: past and present. Onderstepoort J Vet Res 2012; 79:451.

3. Ogawa H, Miyamoto H, Nakayama E, et al. Seroepidemiological prevalence of multiple species of filoviruses in fruit bats (Eidolon helvum) migrating in Africa. J Infect Dis 2015; 212(Suppl 1):101-8.

4. Towner JS, Amman BR, Sealy TK, et al. Isolation of genetically diverse Marburg viruses from Egyptian fruit bats. PLoS Pathog 2009; 5:e1000536.

5. Leroy EM, Kumulungui B, Pourrut X, et al. Fruit bats as reservoirs of Ebola virus. Nature 2005; 438:575-6.

6. Bausch DG, Nichol ST, Muyembe-Tamfum JJ, et al.; International Scientific and Technical Committee for Marburg Hemorrhagic Fever Control in the Democratic Republic of the Congo. Marburg hemorrhagic fever associated with multiple genetic lineages of virus. N Engl J Med 2006; 355:909-19.

7. Adjemian J, Farnon EC, Tschioko F, et al. Outbreak of marburg hemorrhagic fever among miners in Kamwenge and Ibanda districts, Uganda, 2007. J Infect Dis 2011; 204(Suppl):796-9.

8. Leroy EM, Epelboin A, Mondonge V, et al. Human Ebola outbreak resulting from direct exposure to fruit bats in Luebo, Democratic Republic of Congo, 2007. Vector Borne Zoonotic Dis 2009; 9:723-8.

9. Albarino CG, Shoemaker T, Khristova ML, et al. Genomic analysis of filoviruses associated with four viral hemorrhagic fever outbreaks in Uganda and the Democratic Republic of the Congo in 2012. Virology 2013; 442:97-100.

10. Swanepoel R, Smit SB, Rollin PE, et al.; International Scientific and Technical Committee for Marburg Hemorrhagic Fever Control in the Democratic Republic of Congo. Studies of reservoir hosts for Marburg virus. Emerg Infect Dis 2007; 13:1847-51.

11. Le Guenno B, Formenty P, Formentry P, et al. Isolation and partial characterisation of a new strain of Ebola virus. Lancet 1995; 345:1271-4.

12. Georges AJ, Leroy EM, Renaut AA, et al. Ebola hemorrhagic fever outbreaks in Gabon, 1994-1997: Epidemiologic and health control issues. J Infect Dis 1999; 179(Suppl):65-75.

13. Leroy EM, Rouquet P, Formenty P, et al. Multiple Ebola virus transmission events and rapid decline of central African wildlife. Science 2004; 303:387-90.

14. Formenty P, Boesch C, Wyers M, et al. Ebola virus outbreak among wild chimpanzees living in a rain forest of Cote d'Ivoire. J Infect Dis 1999; 179(Suppl 1):120-6.

15. Huijbregts B, Wachter PD, Obiang LSN, Akou ME. Ebola and the decline of gorilla (Gorilla gorilla) and chimpanzee (Pan troglodytes) populations in Minkebe Forest, north-eastern Gabon. Oryx 2003; 37:437-43.

16. Leendertz SAJ, Wich SA, Ancrenaz M, et al. Ebola in great apes-current knowledge, possibilities for vaccination, and implications for conservation and human health. Mammal Review 2017; 47:98-111.

17. Lahm SA, Kombila M, Swanepoel R, Barnes RF. Morbidity and mortality of wild animals in relation to outbreaks of Ebola haemorrhagic fever in Gabon, 19942003. Trans R Soc Trop Med Hyg 2007; 101:64-78. 
18. Morvan JM, Deubel V, Gounon P, et al. Identification of Ebola virus sequences present as RNA or DNA in organs of terrestrial small mammals of the Central African Republic. Microbes Infect 1999; 1:1193-201.

19. Barrette RW, Metwally SA, Rowland JM, et al. Discovery of swine as a host for the Reston ebolavirus. Science 2009; 325:204-6.

20. Allela L, Boury O, Pouillot R, et al. Ebola virus antibody prevalence in dogs and human risk. Emerg Infect Dis 2005; 11:385-90.

21. Rouquet P, Fromont JM, Mermejo M, et al. Wild animal mortality monitoring and human Ebola outbreaks, Gabon and Republic of Congo, 2001-2003. Emerg Infect Dis 2005; 11:283-90.

22. Gonzalez JP, Nakoune E, Slenczka W, Vidal P, Morvan JM. Ebola and Marburg virus antibody prevalence in selected populations of the Central African Republic. Microbes Infect 2000; 2:39-44.

23. Busico KM, Marshall KL, Ksiazek TG, et al. Prevalence of IgG antibodies to Ebola virus in individuals during an Ebola outbreak, Democratic Republic of the Congo, 1995. J Infect Dis 1999; 179(Suppl 1):102-7.

24. Newton-Fisher N, Notman H, Paterson J, Reynolds V, eds. Primates of western Uganda. New York: Springer, 2010.

25. Zhai J, Palacios G, Towner JS, et al. Rapid molecular strategy for filovirus detection and characterization. J Clin Microbiol 2007; 45:224-6.

26. Hume AJ, Ames J, Rennick LJ, et al. Inactivation of RNA viruses by gamma irradiation: a study on mitigating factors. Viruses 2016; 8. doi: 10.3390/v8070204.

27. Gunther S, Feldmann H, Geisbert TW, et al. Management of accidental exposure to Ebola virus in the biosafety level 4 laboratory, Hamburg, Germany. J Infect Dis 2011; 204(Suppl 3):785-90.

28. Falzarano D, Feldmann F, Grolla A, et al. Single immunization with a monovalent vesicular stomatitis virus-based vaccine protects nonhuman primates against heterologous challenge with Bundibugyo ebolavirus. J Infect Dis 2011; 204(Suppl 3):1082-9.

29. Hosmer D, Lemeshow S, Sturdivant R. Applied logistic regression. New York: John Wiley and Sons, 2013.

30. Jacomy M, Venturini T, Heymann S, Bastian M. ForceAtlas2, a continuous graph layout algorithm for handy network visualization designed for the Gephi software. PLoS One 2014; 9:e98679.

31. Shoemaker T, MacNeil A, Balinandi S, et al. Reemerging Sudan Ebola virus disease in Uganda, 2011. Emerg Infect Dis 2012; 18:1480-3.

32. Okware SI, Omaswa FG, Zaramba S, et al. An outbreak of Ebola in Uganda. Trop Med Int Health 2002; 7:1068-75.

33. Khan AS, Toshioko FK, Heymann DL, et al. The reemergence of Ebola hemorrhagic fever, Democratic Republic of the Congo, 1995. J Infect Dis 1999; 179:76-86.
34. Timen A, Koopmans MP, Vossen AC, et al. Response to imported case of Marburg hemorrhagic fever, the Netherland. Emerg Infect Dis 2009; 15:1171-5.

35. Centers for Disease Control and Prevention. Imported case of Marburg hemorrhagic fever - Colorado, 2008. Morb Mortal Wkly Rep 2009; 58:1377-81.

36. Becker S, Feldmann H, Will C, Slenczka W. Evidence for occurrence of filovirus antibodies in humans and imported monkeys: do subclinical filovirus infections occur worldwide? Med Microbiol Immunol 1992; 181:43-55.

37. Leroy EM, Baize S, Volchkov VE, et al. Human asymptomatic Ebola infection and strong inflammatory response. Lancet 2000; 355:2210-5.

38. Richardson ET, Kelly JD, Barrie MB, et al. Minimally symptomatic infection in an Ebola 'hotspot': a cross-sectional serosurvey. PLoS Negl Trop Dis 2016; 10:e0005087.

39. Becquart P, Wauquier N, Mahlakõiv T, et al. High prevalence of both humoral and cellular immunity to Zaire ebolavirus among rural populations in Gabon. PLoS One 2010; 5:e9126.

40. Rowe AK, Bertolli J, Khan AS, et al. Clinical, virologic, and immunologic follow-up of convalescent Ebola hemorrhagic fever patients and their household contacts, Kikwit, Democratic Republic of the Congo. Commission de Lutte contre les Epidemies a Kikwit. J Infect Dis 1999; 179(Suppl 1):28-35.

41. Olival KJ, Islam A, Yu M, et al. Ebola virus antibodies in fruit bats, bangladesh. Emerg Infect Dis 2013; 19:270-3.

42. Sanchez A, Trappier SG, Mahy BW, Peters CJ, Nichol ST. The virion glycoproteins of Ebola viruses are encoded in two reading frames and are expressed through transcriptional editing. Proc Natl Acad Sci U S A 1996; 93:3602-7.

43. Nakayama E, Yokoyama A, Miyamoto H, et al. Enzyme-linked immunosorbent assay for detection of filovirus species-specific antibodies. Clin Vaccine Immunol 2010; 17:1723-8.

44. Albarino CG, Shoemaker T, Khristova M, et al. Genomic analysis of filoviruses associated with four viral hemorrhagic fever outbreaks in Uganda and the Democratic Republic of the Congo in 2012. Virol 2013; 442:97-100.

45. Timen A, Koopmans MP, Vossen AC, et al. Response to imported case of Marburg hemorrhagic fever, the Netherland. Emerg Infect Dis 2009; 15:1171-5.

46. Centers for Disease Control and Prevention. Imported case of Marburg hemorrhagic fever - Colorado, 2008. MMWR Morb Mortal Wkly Rep 2009; 58:1377-81.

47. Bausch DG, Borchert M, Grein T, et al. Risk factors for Marburg hemorrhagic fever, Democratic Republic of the Congo. Emerg Infect Dis 2003; 9:1531-7.

48. Towner JS, Khristova ML, Sealy TK, et al. Marburgvirus genomics and association with a large hemorrhagic fever outbreak in Angola. J Virol 2006; 80: 6497-516. 